\title{
Developing a perioperative protocol for patients having major lower limb amputation: A quality improvement project in conjunction with the Acute Pain Service at Torbay Hospital, UK
}

T Warrener, Department of Anaesthesia and Perioperative Medicine, Torbay Hospital, Torquay, UK $37^{\text {th }}$ ESRA Annual Congress, 12-15 ${ }^{\text {th }}$ September 2018, Dublin, Ireland

\section{Background}

Perioperative mortality following major lower limb amputation (LLA) has come into focus in recent years. Reports from the Vascular Society for Great Britain and Ireland (VSGBI) ${ }^{1}$, and the National Confidential Enquiry into Patient Outcome and Death (NCEPOD) ${ }^{2}$, made several key recommendations on the perioperative care of this high-risk group of patients. Torbay Hospital already uses several well-established perioperative care protocols, and following discussion with colleagues, including the Acute Pain Team, it was felt that a similar protocol for the perioperative management of patients undergoing major LLA would be welcomed. This Quality Improvement (QI) project aims to address the issue of pain control following major LLA.

Many patients undergoing major LLA will have longstanding and often severe ischaemic pain requiring preoperative opioid analgesia. LLA also carries one of the highest incidences of persistent post-surgical pain. Although pre-emptive analgesia has not consistently shown reduction in the incidence of chronic phantom limb pain, pre-operative pain intensity however, has been identified as a significant predictor of chronic phantom limb pain intensity.

Commonly, immediate postoperative analgesic regimes following major LLA include some form of opioid. However, the side effect profile of strong opioids is significant, and later on tolerance, dependence and addiction can all occur with prolonged use. The cost of these opioid-related side effects are well documented.

A multimodal analgesic regime is widely accepted as the gold standard in the management of postoperative pain. Paracetamol, NSAID (if tolerated), plus some form of opioid medication form the backbone of many multimodal regimes. This might be supplemented by RA/LA techniques, antineuropathic agents, and adjuncts (e.g. clonidine, ketamine).

\section{Methods}

This QI project aims to address this issue by using a well-tested QI methodology, the Model for Improvement. This method accelerates improvements in the quality of healthcare processes and outcomes via two phases:

\section{Fundamental questions:}

(i.) What are we trying to accomplish?

Optimal postoperative pain control in patients undergoing major LLA at Torbay Hospital.

\section{(ii.) How will we know that a change is an improvement?}

Improved patient satisfaction/pain scores. Once protocol introduced, repeat process after sufficient period of time to evaluate for change.

\section{(iii.) What changes can we make that will result in improvement?} Introduction of perioperative protocol for patients undergoing major LLA.

2. Plan Do Study Act (PDSA) cycles repeated going forwards to continually evaluate/improve protocol.

\section{Results}

A local data search via the theatres surgical management system revealed 37 cases between March 2016 - March 2018, 12 AKA \& 25 BKA. This is consistent with the National Vascular Registry in terms of case numbers.

Closer inspection of the perioperative management of 14 of these cases (notes review) revealed room for improvement in terms of monitoring standards, type of anaesthesia, consolidation of regional techniques used (broad range), including the use of a perineural catheter, and prescribed post-operative analgesia.
Key findings:

- $64 \%$ performed under GA

- 7 different RA techniques/combinations used

- Only $36 \%$ had catheter technique

- $71 \%$ prescribed antineuropathic agent(s) postoperatively

Documentation of pain scores in patient notes was inconsistent and it was difficult to determine overall patient satisfaction retrospectively. This requires attention with respect to future PDSA cycles.

\section{Conclusions}

In response to the findings highlighted above, an evidence-based perioperative protocol has been produced with input from the Acute Pain Team and RA enthusiasts. Simplicity was considered paramount. The protocol includes notes/helpful tips on the back e.g. 'Ideally all patients should have a catheter technique', recommendation for spinal dosing, RA tips - tunnel catheter, use lock-it dressing/skin glue etc. Following local approval the protocol will be introduced at our institution.

In keeping with the QI methodology outlined above, after sufficient time, subsequent evaluation, in terms of both compliance and outcome, will be undertaken.

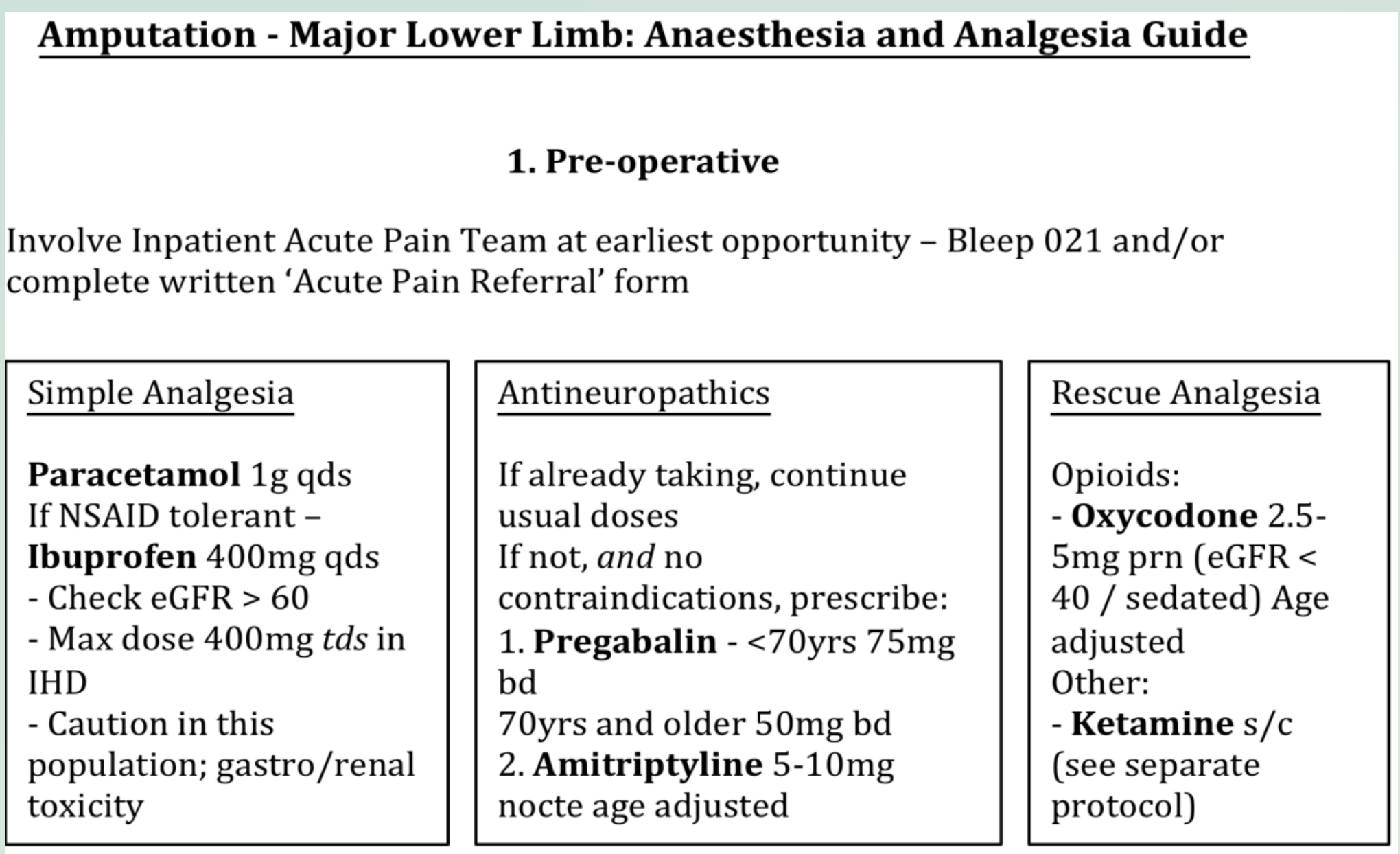

2. Anaesthetic Room/Theatre

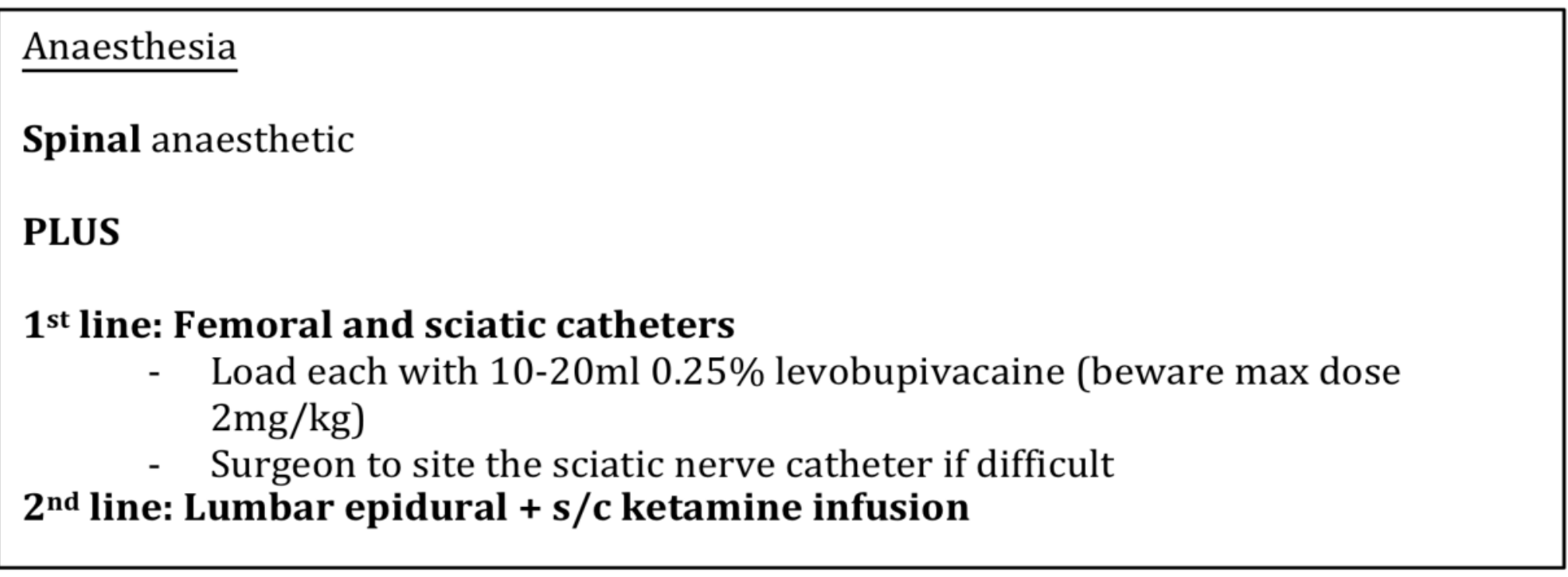

3. Recovery/Post-operative

Handover to Acute Pain Team and inform on-call team if intermittent nerve catheter boluses required

\begin{tabular}{|c|c|c|c|}
\hline$\frac{\text { Simple }}{\text { Analgesia }}$ & & & $\frac{\text { Rescue }}{\text { Analgesia }}$ \\
\hline $\begin{array}{l}\text { Continue as } \\
\text { per Pre- } \\
\text { operative }\end{array}$ & $\begin{array}{l}\text { Continue as per } \\
\text { Pre-operative }\end{array}$ & $\begin{array}{l}\text { Load as above } \\
\text { Prescribe continuous } \\
\text { infusion of } \mathbf{0 . 1 2 5 \%} \\
\text { levobupivacaine at } \mathbf{5} \text { - } \\
\mathbf{1 0 ~} \mathbf{m l} / \mathbf{h r}(\mathrm{start} \text { at } 5 \mathrm{ml}) \\
\text { Max rate is } 14 \mathrm{ml} / \mathrm{hr} \text { in } \\
\text { total }(400 \mathrm{mg} / 24 \mathrm{~h})\end{array}$ & $\begin{array}{l}\text { Consider } \\
\text { Ketamine s/c } \\
\text { (see separate } \\
\text { protocol) } \\
\text { Caution with } \\
\text { opioids }\end{array}$ \\
\hline
\end{tabular}

Acknowledgements

With thanks to Dr Andrea Magides, Dr David Pappin and Dr Andrew McEwen.

References

1. Vascular Society. A Best Practice Clinical Care Pathway for Major Amputation Surgery April 2016.

https://www.vascularsociety.org.uk/ userfiles/pages/files/Resources/ Vasc Soc Amputation Paper V2.pdf

2. National Confidential Enquiry into Patient Outcome and Death (NCEPOD). Lower Limb Amputation: Working Together. 2014

http://www.ncepod.org.uk/2014report2/downloads/WorkingTogetherFullReport.pdf 